\title{
Magnetoencephalographic representation of the sensorimotor hand area in cases of intracerebral tumour
}

\author{
M Oishi, M Fukuda, S Kameyama, T Kawaguchi, H Masuda, R Tanaka
}

J Neurol Neurosurg Psychiatry 2003;74:1649-1654

See end of article for authors' affiliations .....................

Correspondence to: DrM Oishi, Department of Neurosurgery, National Nishi-Niigata Central Hospital, 1-14-1 Masago, Niigata 950-2085, Japan; m.oishi@athena.ocn.ne.jp

Received 4 March 2003 In revised form

17 April 2003

Accepted 25 April 2003

Objective: To assess the clinical value of magnetoencephalography (MEG) in localising the primary hand motor area and evaluating cortical distortion of the sensorimotor cortices in patients with intracerebral tumour.

Methods: 10 normal volunteers (controls) and 14 patients with an intracerebral tumour located around the central region were studied. Somatosensory evoked magnetic fields (SEFs) following median nerve stimulation, and movement related cerebral magnetic fields (MRCFs) following index finger extension, were measured in all subjects and analysed by the equivalent current dipole (ECD) method to ascertain the neuronal sources of the primary sensory and motor components (N2Om and MF, respectively). These ECD locations were defined as the primary hand sensory and motor areas and the positional relations between these two functional areas in controls and patients were investigated.

Results: The standard range of ECD locations of MF to N2Om was determined in controls. In 11 of the 14 patients, MRCFs could identify the primary motor hand area. ECD locations of MF were significantly closer to the $\mathrm{N} 2 \mathrm{Om}$ in the medial-lateral direction in patients than in controls. In patients with a tumour located below the sensorimotor hand area, relative ECD locations of MF to $\mathrm{N} 20 \mathrm{~m}$ moved anteriorly over the standard range determined in the control subjects. These MEG findings correlated well with radiological tumour locations. The mean estimated ECD strength of MF was significantly lower in patients than in controls.

Conclusions: MRCF was useful in localising the primary motor hand area in patients with intracerebral tumour. The relative ECD locations of $M F$ to $\mathrm{N} 20 \mathrm{~m}$ describe the anatomical distortion of the sensorimotor cortex.

E arly studies based on direct electrical stimulation of the cerebral cortices of awake neurosurgical patients established the somatotopic arrangement of the human motor and sensory cortices. ${ }^{1}$ Recent neuroimaging modes such as functional magnetic resonance imaging (fMRI), ${ }^{2-7}$ positron emission tomography (PET), ${ }^{89}$ and magnetoencephalography $(M E G)^{10-17}$ have allowed the non-invasive identification of these functional areas and have confirmed the previously described sensory and motor homunculi. The hand area of each homunculus has been examined in relation to the anatomically characteristic landmark "knob" struc-

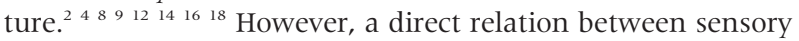
and motor hand areas has never been described.

MEG is a non-invasive way of measuring extracranial magnetic fields generated by intraneuronal electric currents. ${ }^{19}$ As magnetic fields are relatively unaffected by the different electrical conductivities of the brain, cerebrospinal fluid, skull, and skin, MEG can accurately localise the origin of intraneuronal electric currents that contribute to extracranial magnetic fields by fitting to an equivalent current dipole (ECD) model. ${ }^{20}$ The accuracy of MEG localisation has been shown to be within a few millimetres of that obtained by direct cortical mapping. ${ }^{21}$ The clinical value of MEG has been reported mainly in the identification of somatosensory, auditory, and visual areas in neurosurgical patients. ${ }^{11-13} 15$ 22-24 For the identification of the primary motor hand area by MEG, the movement related cerebral magnetic field (MRCF) generated by spontaneous finger movements is used. ${ }^{25-27}$ However, only a few investigators have reported clinical applications of the MRCF, mainly in patients with brain tumours. ${ }^{1728-30}$

In the present study, our main aim was to clarify the clinical ability of MEG to evaluate the cortical distortion of sensorimotor areas in patients with intracerebral tumours. To do this, we first determined the standard positional relation between human hand motor and sensory areas using MEG in normal subjects. We then recorded MRCFs from the patients with intracerebral tumours and evaluated whether the MEG relation between hand motor and sensory areas corresponded to the anatomical findings in these patients.

\section{METHODS}

\section{Subjects}

Ten right handed normal volunteers (eight men, two women; age 20 to 41 years, mean 31.5 ) and 14 patients with intracerebral tumours adjacent to the sensorimotor area ( six men, eight women; age 20 to 66 years, mean 38.6) participated in the study. Patients were evaluated for surgical treatment between June 2000 and January 2002. Thirteen of the 14 patients were treated surgically. The final pathological diagnoses in these 13 patients were glial tumour $(n=8)$, cavernous angioma $(n=4)$, and metastatic tumour $(n=1)$. The remaining patient with radiologically diagnosed cavernous angioma did not undergo surgery. Informed consent was obtained from all controls and patients after the experimental procedures were explained.

\section{Magnetoencephalography}

MEG recording was done in a magnetically shielded room at the National Nishi-Niigata Central Hospital, Niigata, Japan. A

\footnotetext{
Abbreviations: $E C D$, equivalent current dipole; $M E G$,

magnetoencephalography; MRCF, movement related cerebral magnetic field; SEF, somatosensory evoked magnetic field
} 
commercially available helmet shaped neuromagnetometer (Neuromag 204; 4D-Neuroimaging, San Diego, California, USA) consisting of 204 planar-type gradiometers was used. This gradiometer configuration is known to detect the largest signal at the site on the head immediately above the current source. ${ }^{31}$ Each subject was placed in a comfortable sitting position. Head position with respect to the helmet was determined using four indicator coils placed on the scalp that were referenced to three anatomical fiducial markers (nasion and bilateral preauricular points). The recorded data were analysed by fitting each component to a single ECD model in the three dimensional reference frame determined by the fiducial markers. ECDs with a goodness of fit better than $90 \%$ were accepted and were superimposed onto the patient's three dimensional brain magnetic resonance (MR) images obtained with a 1.5 Tesla system (MAGNEX Epios15; Shimazu, Kyoto, Japan).

\section{Somatosensory evoked magnetic fields}

A median nerve was stimulated sequentially with an electrical square wave of $0.2 \mathrm{~ms}$ duration and $2.8 \mathrm{~Hz}$ repetition rate. The $350 \mathrm{~ms}$ period of a total of 200 epochs, including a $50 \mathrm{~ms}$ prestimulus period for baseline setting, was averaged. Data were sampled at $1000 \mathrm{~Hz}$ with the low pass filter set at $300 \mathrm{~Hz}$ and analysed with the high pass filter set at $10 \mathrm{~Hz}$. The primary sensory area of the median nerve was determined by localisation of an ECD for N20m, which is a well known component indicating the earliest cortical response after stimulus. ${ }^{32}$

\section{Movement related cerebral magnetic fields}

The standard method for recording MRCFs has been described in detail elsewhere. ${ }^{27}$ We modified the method

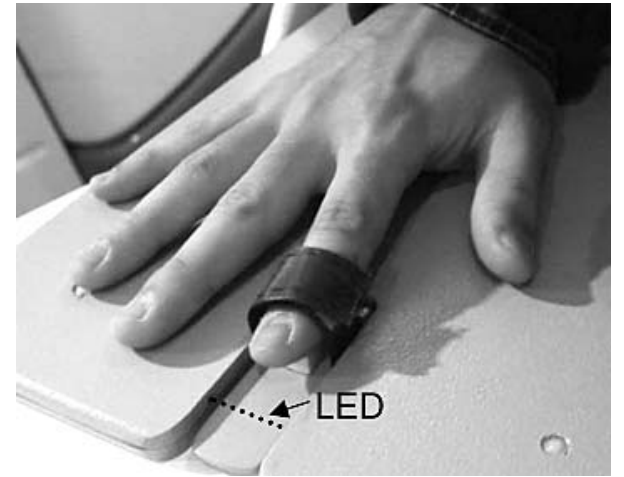

Figure 1 The finger movement pad. When the small plate fixed to the index finger cuts off the light emitting diode because of finger extension, an input is triggered.

using a specific trigger board (finger movement pad; 4Dneuroimaging) (fig 1). The input is triggered by this board and is averaged when the subject extends the index finger appropriately. Each subject was instructed to move the finger at self paced intervals of about five seconds with a sharp movement that begins after the finger muscles are totally relaxed, and to avoid blinking and other eye movements during the task. A total of 70 to 100 epochs and the $1000 \mathrm{~ms}$ prestimulus and $1000 \mathrm{~ms}$ poststimulus periods were averaged. The first $200 \mathrm{~ms}$ of the prestimulus period was used to obtain baseline data. Data were sampled at $500 \mathrm{~Hz}$ with the low pass filter set at $160 \mathrm{~Hz}$ and analysed with the bandpass filter set between 1 and $35 \mathrm{~Hz}$. The MRCF waveform consists of several major components (fig 2) for which functional
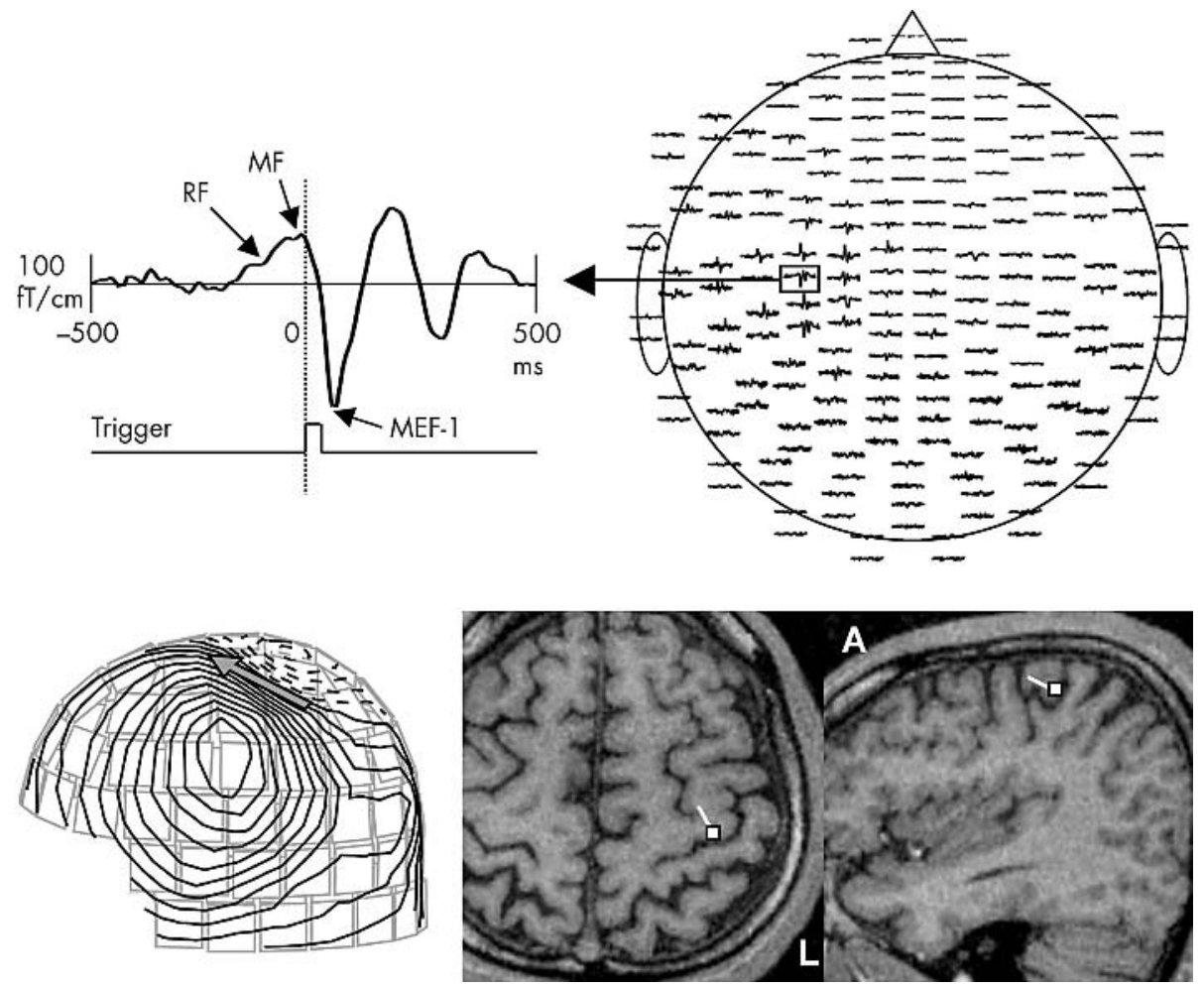

Figure 2 Identification of the motor hand area by the movement related cerebral magnetic field (MRCF) in a normal volunteer. Upper panels: a typical waveform (left) and whole scalp waveforms (right) of MRCF in response to a right index finger extension task. RF, readiness field; MF, motor field; MEF1, movement evoked field 1. Lower left panel: isocontour map of the MF shows a clear dipole pattern. Lower middle and right panels: MF dipole superimposed onto axial (middle) and sagittal (right) MR images allows estimation of the neuronal source at the characteristic structure in the anterior wall of the central sulcus. The square and tail represent the dipole location and orientation, respectively. 
significance has been postulated in previous reports..$^{25-27}$ Of these components, we used an ECD for the motor field (MF), which is considered to reflect the final corticospinal outflow, to determine the primary motor area. ${ }^{25-27}$

\section{Evaluation of the primary motor hand area}

We use the ECD for N20m elicited by median nerve stimulus as the primary sensory hand area and the ECD for MF elicited by the index finger extension task as the primary motor hand area. In addition, we used the ECD for N20m as a reference to assess the relative locations of the MF, because detection of somatosensory evoked magnetic fields (SEFs) is the established method, and N20m provides quite accurate source localisation. ${ }^{1124} 32$

We investigated the distribution of the ECD locations of MF relative to those of $\mathrm{N} 20 \mathrm{~m}$ in the controls to determine the standard range of the relation between the sensory and motor hand areas. The grand mean $( \pm 2 \mathrm{SD})$ of the MF location was used as the standard range in each of three dimensional axes. To assess tumour related cortical distortion in sensorimotor areas, the standard range was applied to each patient, and the relative location of MF to $\mathrm{N} 20 \mathrm{~m}$ was compared with that in the control subjects. We also investigated the appearance of the central regions on MR images in relation to the MEG derived MF locations. ECD strength, which is considered to reflect cortical activity, was calculated in all subjects. Mean ECD strength of patients was compared with that of controls. Welch's $t$ test was used for statistical comparisons. A probability (p) value of $<0.05$ was considered significant.

\section{RESULTS}

Control study in normal volunteers

The ECDs for N20m in all volunteers were located in the posterior wall of the central sulcus at the lateral side of the knob structure, with anterior superior medial orientation. ECDs for the MF were located in the anterior wall of the central sulcus just at the knob structure, with anterior superior medial orientation. The relative ECD locations of MF to $\mathrm{N} 20 \mathrm{~m}$ in the 10 normal volunteers are shown in fig $3 \mathrm{~A}$. The grand mean (2SD) MF locations were 12.3 (3.4) mm medial to, $1.8(2.8) \mathrm{mm}$ posterior to, and $2.6(3.1) \mathrm{mm}$ superior to the $\mathrm{N} 20 \mathrm{~m}$ locations. All 10 locations were within the standard range. The mean dipole strength was 14.8 (4.0) nAm.

\section{MRCF results in patients}

MRCFs were obtained in 11 of the 14 patients (79\%); two older patients and one patient with mild hemiparesis were excluded. The relative ECD locations of MF to N20m in these 11 patients are shown in fig 3B. The medial-lateral distances of ECD locations between MF and N20m were significantly shorter in these patients than in the controls $(p<0.05)$. There was no significant difference in either anterior-posterior or superior-posterior distance. The ECDs for MF lay outside of the standard range in four patients and within the standard
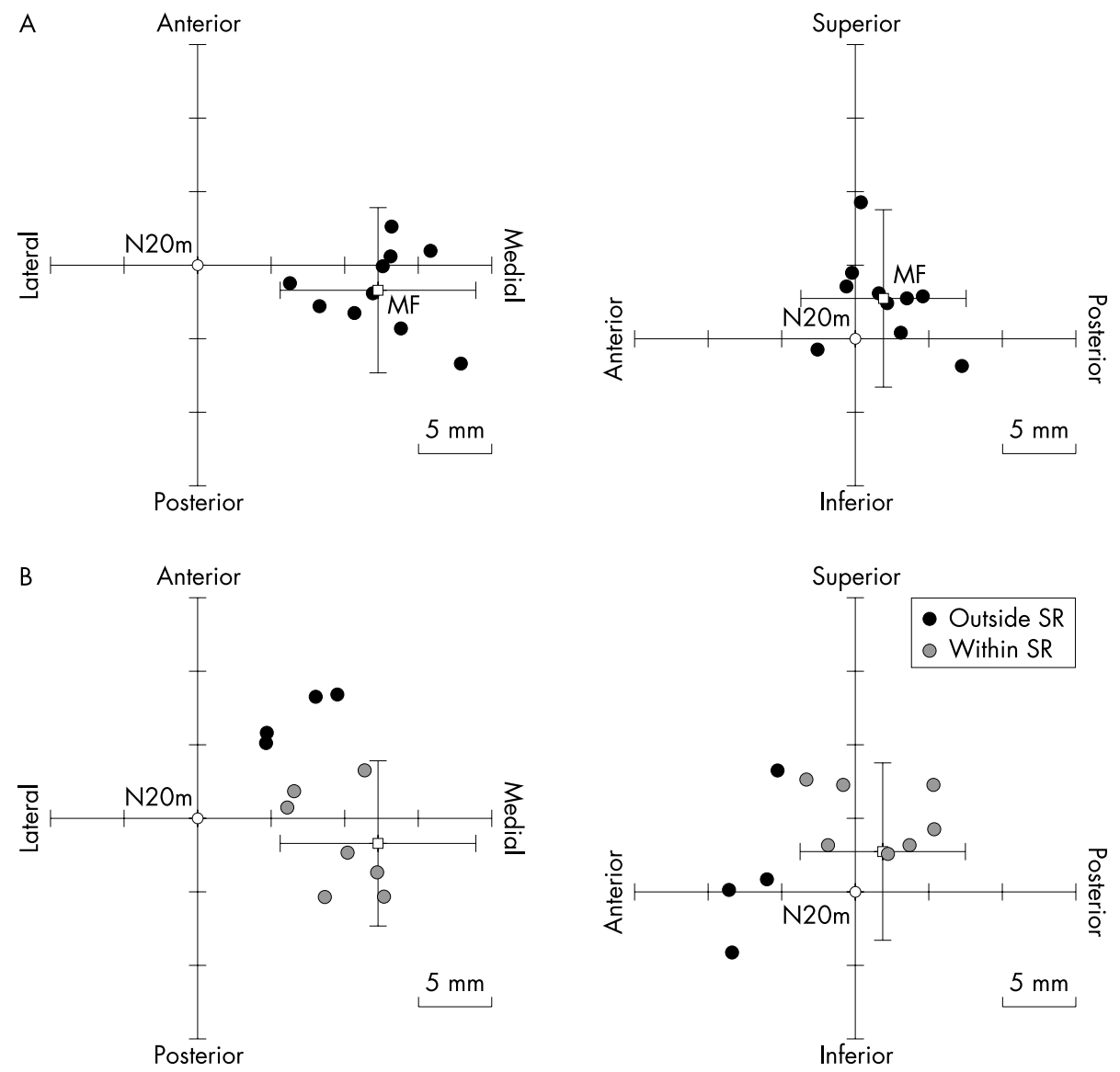

Figure 3 (A) The equivalent current dipole (ECD) locations of MF in relation to those of N20m in controls. ECD locations of all subjects are shown as black dots, and the mean ECD location is shown as a white square with 2 SD error bars. ECDs of all subjects were included within the grand mean $\pm 2 S D$ in each direction, which is taken as the standard range. (B) The equivalent current dipole (ECD) locations of MF in relation to those of N2Om in patients. Grey and black dots indicate the location within and outside the standard range, respectively. In the medial-lateral direction, ECD locations between MF and N2Om are significantly closer than those in controls $(p<0.05)$. The ECDs are distributed anteriorly outside the standard range in four patients. SR, standard range. 
range in seven. MF locations lying outside of the standard range were distributed significantly more anteriorly than those lying within the standard range $(\mathrm{p}<0.005)$. Differences in the medial-lateral and superior-inferior directions did not achieve statistical significance. A schema of the tumour locations determined on MR images is presented in fig 4. In all four patients whose ECDs lay outside of the standard range, the tumours were located just beneath the sensorimotor cortices. However, in the patients whose ECDs lay within the standard range, the tumours lay anteriorly or posteriorly remote from the sensorimotor cortices. Mean dipole strength in the patients was 11.4 (3.5) nAm, significantly lower than in the volunteers $(\mathrm{p}<0.05)$.

\section{Intraoperative monitoring and surgical outcomes}

Total or partial removal of tumours was undertaken in eight of the 11 patients in whom MRCFs were obtained. Two of the three remaining patients underwent stereotactic biopsy to confirm the pathological diagnosis, and one is still being observed. Intraoperative motor evoked potential (MEP) monitoring was done in five of the eight patients who underwent tumour removal surgery. In all five of these patients, cortical stimulation at the sites anatomically coinciding with the MF sources evoked myoelectrographic activities of the contralateral thenar muscle. Surgical outcome was good; no patient experienced a long lasting neurological deficit corresponding to a sensorimotor cortical area.

\section{CASE ILLUSTRATIONS}

A patient with the ECD lying outside of the standard range is shown in fig 5 . This 20 year old woman felt slight right hand clumsiness which completely disappeared a few days later. Radiological examination showed a haemorrhagic lesion in the central region, indicating a cavernous angioma. On MR
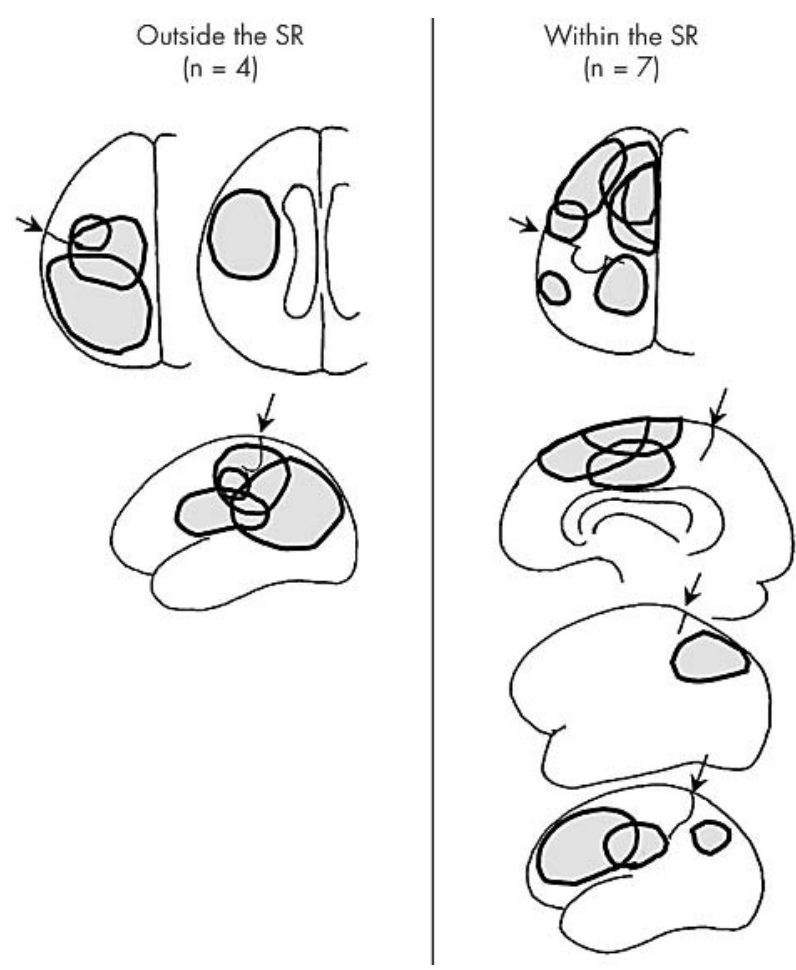

Figure 4 Schemas showing tumour locations in patients outside and within the standard range (SR). The sensorimotor areas were compressed by tumours locating below in the former patients and by tumours locating anteriorly or posteriorly in the latter. images, the sensorimotor cortices appeared to be compressed by the lesion just beneath the central sulcus. MEG also showed the anterior-posterior distance between MF and $\mathrm{N} 20 \mathrm{~m}$ locations to be beyond the standard range. This patient is still being observed and has not undergone surgery.

A patient with the ECD lying within the standard range is shown in fig 6. This 58 year old man experienced only one seizure with short periods of right hemiconvulsion. MRI revealed an intracerebral tumour in the left superior frontal region. MEG was used to determine sensory and motor hand areas, and the tumour was confirmed to extend to the precentral gyrus. The sensorimotor hand area was compressed laterally by the tumour, and the MF-N20m distance in the medial-lateral direction was found to lie within the standard range but just at the lower limit. The tumour was removed completely with the aid of intraoperative SEP and MEP monitoring. After surgery, the patient experienced severe hemiparesis but recovered completely within one week.

\section{DISCUSSION}

Advanced minimally invasive neuroimaging techniquessuch as fMRI, PET, and MEG-are available for presurgical functional mapping. The usefulness of these methods has been validated in previous comparative intraoperative mappings and surgical studies. Each method has its own advantages and limitations, based on principles of operation, measurement methods, or cost restraints. While MEG reflects direct neuronal activity, ${ }^{19}$ the other methods rely on haemodynamic (fMRI) or metabolic (PET) correlates of neuronal activity. The greatest advantage of MEG for functional mapping in patients with intracranial lesions is that it does not have to take into account the influence on the blood supply or metabolism of the space occupying lesion itself or the perilesional oedema that can lead to incorrect functional localisation. ${ }^{5712293033}$ Moreover, the excellent temporal resolution of MEG-similar to that of electroencephalography - is one of MEG's advantages and permits the identification of the primary cortical response to various stimuli.

To investigate the cerebral activity that occurs during motor execution, Kornhuber and Deecke recorded movement related cortical potentials elicited by a finger movement task in $1965 .{ }^{34}$ Some investigators have recently used MEG to identify generators of pre- and post-movement cerebral activities..$^{25-27}$ In response to unilateral finger movement, MRCF waveforms are recorded mainly over the contralateral central region and have some well known components. Readiness field (RF) - the slow slope increasing from 0.5 to $2 \mathrm{~s}$ before movements-reflects the planning involved in voluntary movements. MF is the peak following RF just before movement onset, and reflects primary motor activation. Movement related evoked field-1 (MEF-1) is the largest and most stable peak in the MRCF waveform and occurs 80 to $100 \mathrm{~ms}$ after the movement. Previous investigators have applied this component to clinical settings as the target response to identify the primary motor area. ${ }^{28}{ }^{30}{ }^{33}$ However, MEF-1 is not likely to directly reflect primary motor function owing to its latency. It is considered to reflect a certain kind of sensory activation related to the movement itself. ${ }^{25-27}$ In MEG analysis, selection of the target response is very important for accurate functional localisation. In the present study, we used MF as the most reliable target to evaluate primary motor function and obtained satisfactory localisations in both controls and patients. On the other hand, we could not obtain a useful MF component in two older patients and one hemiparetic patient. Averaging of MRCF responses requires appropriately repetitive finger movements as the trigger, which can sometimes be difficult for such 

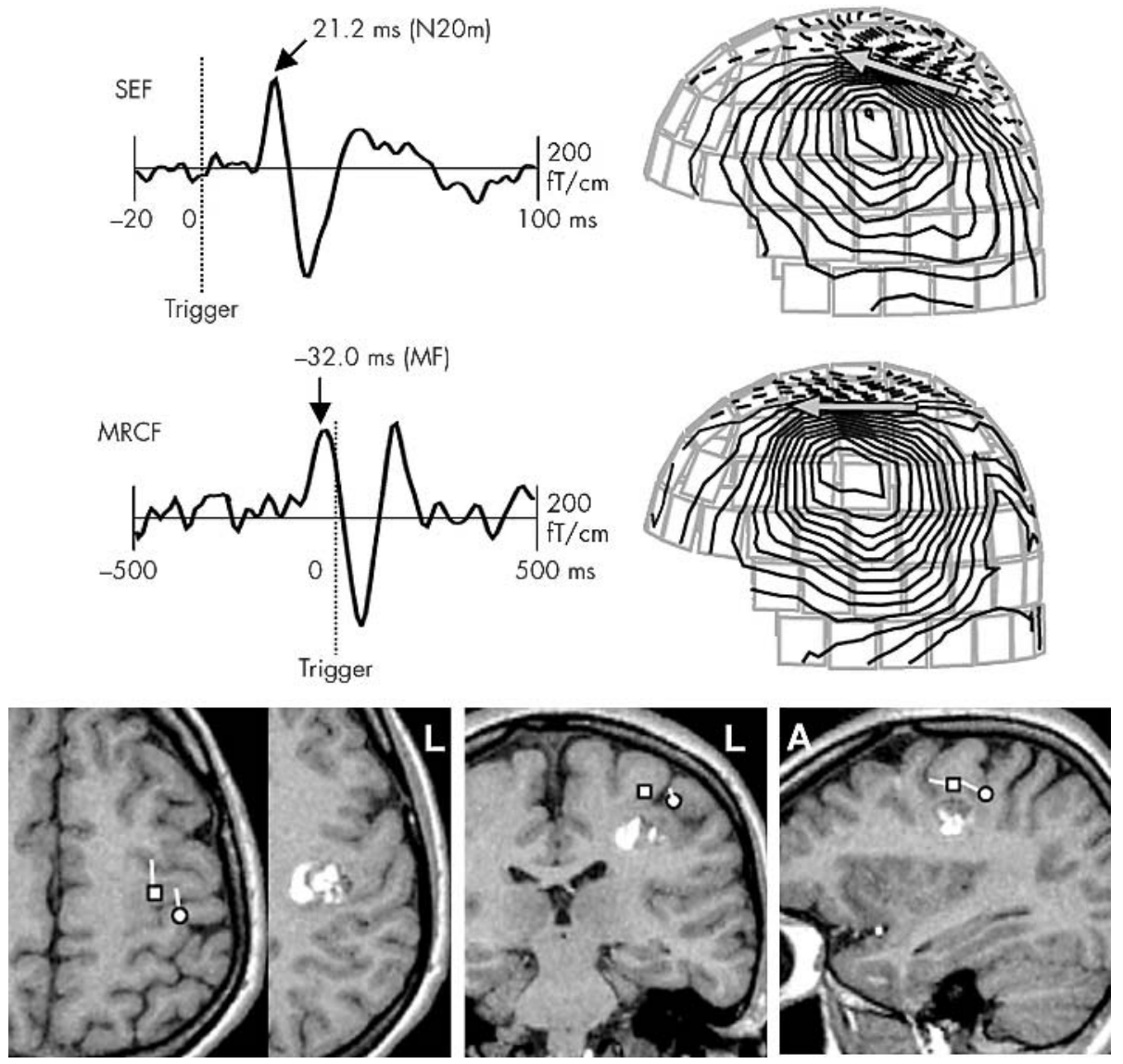

Figure 5 Data from a typical case lying outside the standard range. Upper panels: a typical somatosensory evoked magnetic field waveform from the left central region in response to the right median nerve stimulation (leff) and the isocontour map of N20m at $21.2 \mathrm{~ms}$ after stimulus (right). Middle panels: a typical movement related cerebral magnetic field waveform from the left central region in response to the right index finger extension task (left) and the isocontour map of MF at 32.0 ms before the task (right). Lower panels: axial (left), coronal (middle), and sagittal (right) T1 weighted magnetic resonance images with superimposed ECDs for N2Om and MF. Circle and square represent ECD locations of N2Om and MF, respectively. Tails represent the ECD orientations. The ECDs are separated in the anterior-posterior direction by tumour compression from just beneath.

patients. In particular, hemiparetic patients often have a tumour located close to the sensorimotor cortices, which need precise presurgical functional mapping. Further improvement in the MRCF recording technique is still expected.

In the controls, we verified the standard relation between human motor and sensory hand areas by MEG. Human motor and sensory cortices face each other anatomically, but locations of the hand area in both cortices have a definite relation in distance and direction. The characteristic structure of the "precentral knob" in the anterior wall of the central sulcus, which is shaped like an omega or epsilon character in the MR axial plane and like a hook in the sagittal plane, has been reported to contain the motor hand functions. ${ }^{2} 4{ }^{9}{ }^{18}{ }^{27} \mathrm{It}$ has been confirmed that the location of the sensory hand area is at the lateral portion of the "knob" in the posterior wall of the central sulcus. ${ }^{11}{ }^{12} 16{ }^{24}$ In our study, the standard range of MF locations referenced to N20m coincided completely with this previous knowledge and accurately represented the relation between these two functional areas.

Relative ECD locations of MF to N20m also successfully represented the degree and direction of cortical distortion of the sensorimotor areas in patients with brain tumours. In the medial-lateral direction, ECD locations of MF were
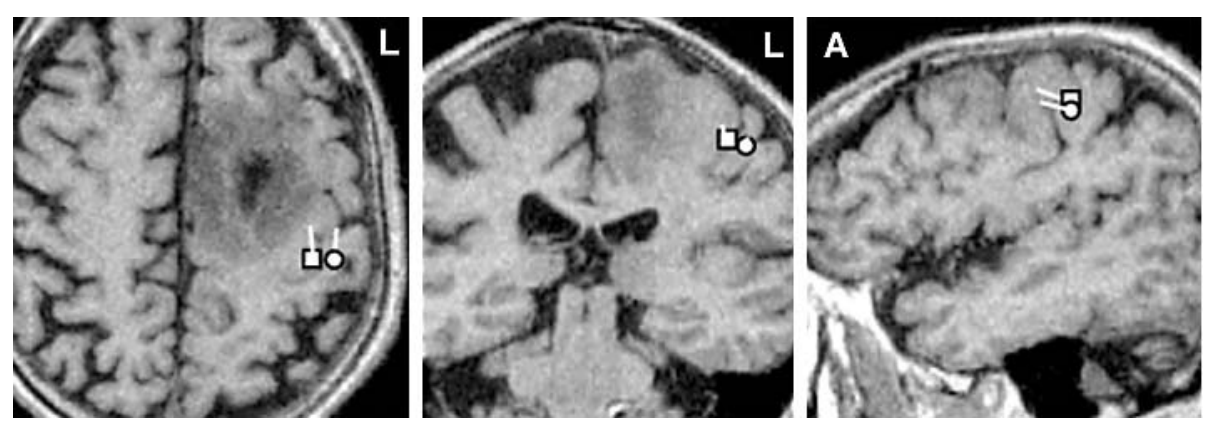

Figure 6 Data from a typical case lying within the standard range. Axial (left), coronal (middle), and sagittal (right) T1 weighted magnetic resonance images with superimposed ECDs of N2Om and MF. The ECDs are moved together in the posterior direction by tumour compression. 
significantly closer to the $\mathrm{N} 20 \mathrm{~m}$ sources in patients than in controls. This suggests that the distance between the sensory and motor hand areas tends to be shortened in the mediallateral direction by tumours located even in regions remote from the central sulcus. In regard to the anterior-posterior direction, we found four patients with ECDs lying outside of the standard range anteriorly, based on relative ECD locations of MF to $\mathrm{N} 20 \mathrm{~m}$. We assume that the sensory and motor hand areas in these patients were compressed from just beneath the central sulcus by the tumours and were separated in the anterior-posterior direction. Indeed, MR images displayed brain tumours located just beneath the central sulcus in those patients. However, in patients with ECDs within the standard range, the brain tumours were located in the clearly anterior or posterior region remote from the central sulcus. These results suggest that magnetoencephalographic findings correlate with anatomical findings of tumour location and compression of the cortex.

It may be possible to use estimated ECD strength to evaluate primary motor function quantitatively. ${ }^{35}$ Evaluation in each patient is still preliminary, but the mean ECD strength in patients was significantly smaller than in controls. We did not obtain a correlation between the degree of motor weakness and ECD strength because the patients in this study had no, or only slight, paresis. However, ECD strength may be useful in evaluating motor function of patients with lesions in the central region, even when the patients do not present with hemiparesis.

We conducted the present study with a view to maximal removal of the tumour without any resulting functional deficits, particularly hand paresis. In the present series, MEP by direct cortical stimulation confirmed the accuracy of MEG in five patients in whom both MRCFs and MEPs were obtained. With the exception of a patient with postoperative transient paresis after resection involving a supplementary motor area, no patient experienced any resulting deficit from surgery. Intraoperatively, the cortical SEP is the gold standard for defining the central sulcus and easily identifying the primary somatosensory cortex. ${ }^{36}$ In addition, accurate determination of the relation between the motor and sensory hand areas by MEG can be helpful in presurgical planning and can result in good surgical outcomes without functional deficits.

\section{ACKNOWLEDGEMENTS}

We thank Kuniko Tsuchiya and Tohru Kondo for their technical assistance and Dr Nobuyuki Nishitani of the Research Institute of National Rehabilitation Centre for the Disabled for his instruction in MRCF measurement.

\section{Authors' affiliations}

M Oishi, S Kameyama, H Masuda, Department of Neurosurgery, National Nishi-Niigata Central Hospital, Niigata, Japan

M Fukuda, T Kawaguchi, R Tanaka, Department of Neurosurgery, Brain Research Institute, Niigata University

Competing interests: none declared

\section{REFERENCES}

1 Penfield W, Boldrey E. Somatic motor and sensory representation in the cerebral cortex of man as studied by electrical stimulation. Brain 1937;60:389-443.

2 Breisteiner R, Windischberger $C$, Lanzenberger $R$, et al. Finger somatotopy in human motor cortex. Neuroimage 2001;13:1016-26.

3 Fandino J, Kollias SS, Wieser HG, et al. Intraoperative validation of functional magnetic resonance imaging and cortical reorganization pattern in patients with brain tumors involving the primary motor cortex. J Neurosurg 1999:91:238-50

4 Hlustik P, Solodkin A, Guallapalli RP, et al. Somatotopy in human primary motor and somatosensory hand representations revisited. Cereb Cortex $2001 ; 11: 312-21$

5 Inove T, Shimizu H, Nakasato N, et al. Accuracy and limitation of functional magnetic resonance imaging for identification of the central sulcus: comparison with magnetoencephalography in patients with brain tumors. Neuroimage 1999;10:738-48.

6 Lehericy S, Duffau H, Cornu P, et al. Correspondence between functional magnetic resonance imaging somatotopy and individual brain anatomy of the central region: comparison with intraoperative stimulation in patients with brain tumors. J Neurosurg 2000;92:589-98.

7 Schreiber A, Hubbe U, Ziyeh S, et al. The influence of gliomas and nonglial space-occupying lesions on blood-oxygen-level-dependent contrast enhancement. Am J Neuroradiol 2000;21:1055-63.

8 Bittar RG, Olivier A, Sadikot AF, et al. Cortical motor and somatosensory representation: effect of cerebral lesions. J Neurosurg 2000;92:242-8.

9 Boling W, Olivier A, Bittar RG, et al. Localization of hand motor activation in Broca's pli de passage moyen. J Neurosurg 1999;91:903-10.

10 Cheyne D, Kristeva R, Deecke L. Homuncular organization of human motor cortex as indicated by neuromagnetic recordings. Neurosci Lett 1991; 122:17-20

11 Kawamura T, Nakasato N, Seki K, et al. Neuromagnetic evidence of pre- and post-central cortical sources of somatosensory evoked responses. Electroencephalogr Clin Neurophysiol 1996;100:44-50.

12 Kumabe T, Nakasato N, Inove T, et al. Primary thumb sensory cortex located at the lateral shoulder of the inverted omega-shape on the axial images of the central sulcus. Neurol Med Chir (Tokyo) 2000;40:393-403.

13 Nagamatsu K, Nakasato N, Hatanaka K, et al. Neuromagnetic localization of N15, the initial cortical response to lip stimulus. Neuroreport $2001 ; 12: 1-5$.

14 Nakamura A, Yamada T, Goto A, et al. Somatosensory homunculus as drawn by MEG. Neuroimage 1988;7:377-86.

15 Nakasato N, Yoshimoto T. Somatosensory, auditory, and visual evoked magnetic fields in patients with brain diseases. J Clin Neurophysiol 2000;17:201-11.

16 Ohtomo S, Nakasato N, Kawamura T, et al. Correspondence of anatomy and function in the human digit sensory cortex revealed by an MRI-linked wholehead MEG system. Electroencephalogr Clin Neurophysiol 1996;47(suppl):91-5

17 Oishi M, Kameyama S, Watanabe M, et al. Presurgical functional mapping of the sensorimotor area using evoked magnetic fields. No Shinkei Geka 2002;30:391-7.

18 Yousry TA, Schmid UD, Alkadhi H, et al. Localization of the motor hand area to a knob on the precentral gyrus. A new landmark. Brain 1997;120:141-57.

19 Cohen D. Magnetoencephalography: detection of the brain's electrical activity with a superconducting magnetometer. Science 1972;175:664-6.

20 Barth DS, Suthering WW, Engel J, et al. Neuromagnetic localization of epileptiform spike activity in the human brain. Science 1982;218:891-4

21 Gallen CC, Schwartz BJ, Bucholz RD, et al. Presurgical localization of functional cortex using magnetic source imaging. J Neurosurg 1995;82:988-94.

22 Alberstone CD, Skirboll SL, Benzel EC, et al. Magnetic source imaging and brain surgery: presurgical and intraoperative planning in 26 patients. J Neurosurg 2000;92:79-90.

23 Nakasato N, Kumabe T, Kanno A, et al. Neuromagnetic evaluation of cortical auditory function in patients with temporal lobe tumors. J Neurosurg 1997:86:610-18.

24 Sobel DF, Gallen CC, Schwartz BJ, et al. Locating the central sulcus: comparison of MR anatomic and magnetoencephalographic functional methods. Am J Neuroradiol 1993;14:915-25.

25 Cheyne D, Weinberg $\mathrm{H}$. Neuromagnetic fields accompanying unilateral finger movements: pre-movement and movement-evoked fields. Exp Brain Res 1989;78:604-12.

26 Hoshiyama $M$, Kakigi R, Berg $P$, et al. Identification of motor and sensory brain activities during unilateral finger movement: spatiotemporal source analysis of movement-associated magnetic fields. Exp Brain Res 1997; 115:6-14

27 Kristeva-Feige R, Rossi S, Feige B, et al. The bereitschaftspotential paradigm in investigating voluntary movement organization in humans using magnetoencephalography (MEG). Brain Res Brain Res Protocol 1997:1:13-22.

28 Ganslandt O, Fahlbusch R, Nimsky C, et al. Functional neuronavigation with magnetoencephalography: outcome in 50 patients with lesions around the motor cortex. J Neurosurg 1999;91:73-9.

29 Kober H, Nimsky C, Möller M, et al. Correlation of sensorimotor activation with functional magnetic resonance imaging and magnetoencephalography in presurgical functional imaging: a spatial analysis. Neuroimage 2001;14:1214-28.

30 Nimsky C, Ganslandt O, Kober $\mathrm{H}$, et al. Integration of functional magnetic resonance imaging supported by magnetoencephalography in functional neuronavigation. Neurosurgery 1999;44:1249-56.

31 Knuutila JE, Ahonen Al, Hämäläinen MS, et al. A 122-channel whole-cortex SQUID system for measuring the brain's magnetic fields. IEEE Trans Magn 1993:29:3315-20.

32 Kakigi R. Somatosensory evoked magnetic fields following median nerve stimulation. Neurosci Res 1994;20:165-74.

33 Stippich C, Freitag P, Kassubek J, et al. Motor, somatosensory and auditory cortex localization by FMRI and MEG. Neuroreport 1998:9:1953-7.

34 Kornhuber HH, Deecke L. Hirnpotenrialänderungen bei Willkürbewegungen und passiven Bewegungen des Menschen: Bereitschaftpotential und reafferente Potentiale. Pflügers Arch 1965;284:1-17.

35 Taniguchi M, Yoshimine T, Cheyne D, et al. Neuromagnetic fields preceding unilateral movements in dextrals and sinistrals. Neuroreport 1998:9:1497-502

36 Wood CC, Spencer DD, Allison T, et al. Localization of human sensorimotor cortex during surgery by cortical surface recording of somatosensory evoked potentials. J Neurosurg 1988;68:99-111. 